\title{
Using In-Situ TEM to Investigate the Role of Lithium Iodide Addition to Lithium Thiophosphate
}

Nikhilendra Singh ${ }^{1}$, James Horwath ${ }^{2}$, Timothy Arthur ${ }^{1}$, Daan Hein Alsem ${ }^{3}$, Marm Dixit ${ }^{4}$, Pavel Shevchenko ${ }^{5}$, Michael Jones ${ }^{1}$, Kelsey Hatzell ${ }^{4}$ and Eric Stach $^{2}$

${ }^{1}$ Toyota Research Institute of North America, United States, ${ }^{2}$ University of Pennsylvania, Philadelphia, Pennsylvania, United States, ${ }^{3}$ Hummingbird Scientific, Lacey, Washington, United States, ${ }^{4}$ Vanderbilt University, United States, ${ }^{5}$ Argonne National Laboratory, United States

To keep up with consumer demand for electric and hybrid vehicles, energy storage devices with higher energy and power densities, as well as higher cycle life, are needed. To meet this need, Li metal anode batteries have recently presented themselves as ideal candidates with maximum theoretical specific capacity ten-fold that of commercially used anodes like graphite. Liquid Li-sulfur is one battery system that has come to the forefront in this research field because of its high theoretical capacity and low cost.

However, this material system can display polysulfide dissolution and electrolyte decomposition, which can inhibit the performance and the durability of these systems in practice. Several approaches to solve these issues have been taken. Using solid electrolytes is one such approach that also has the added benefit that the solid electrolyte can form a mechanical barrier to prevent Li dendrite formation. Still, solid electrolytes have lower ionic conductivities, and their interfacial stability at the Li metal interface is low. While research into new solid electrolytes keeps improving their ionic conductivities, research into the processes at the interface of sulfide-based solid-state electrolytes and $\mathrm{Li}$ metal are rare. As such, the understanding of these interfacial processes is crucial to achieving solid-state Li-metal batteries.

In this study, we present research into the electrochemical properties of sulfide-based solid-state electrolytes in contact with Li metal anodes. We use ex-situ X-ray Tomography and in-situ transmission electron microscopy to show the interfacial processes and failure modes between Li metal and lithium thiophosphates. Furthermore, we studied the deposition and dissolution processes of Li metal from these electrolytes and the effect of Li cycling on the electrolyte structure in the presence/absence of lithium iodide. Using a TEM biasing sample holder with a movable electrical probe (Figure 1), we were able to make a very localized electrical contact to a sample inside the TEM to study these effects (Figure 2). This method was previously successfully applied to study a range of other materials systems [1-4]. The presented work enables a comparison of Li deposition and dissolution properties below and above the critical current densities for each lithium thiophosphate electrolyte material $[5,6]$. From these results, we can deduce the critical factors governing the interfacial, morphological, and failure evolution mechanisms during Li cycling in these battery systems. 

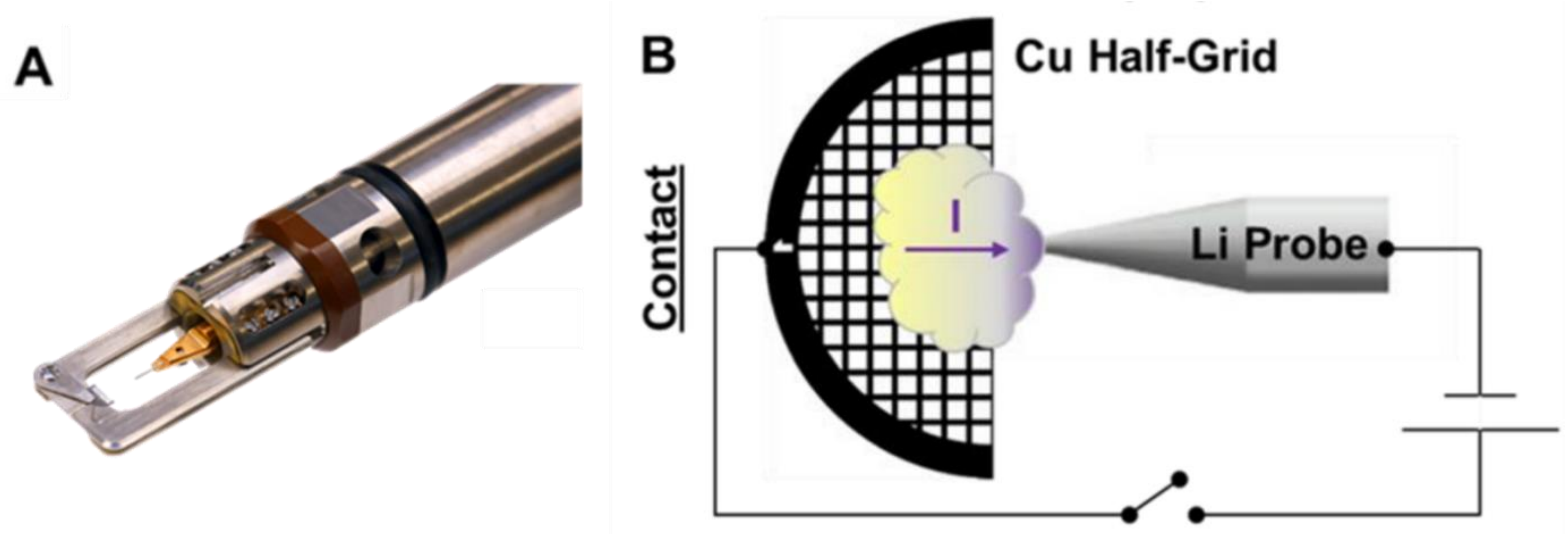

Figure 1. Figure 1: A) tip of the operando TEM biasing sample holder with movable probe; B) schematic of the experiment showing the Li metal anode probe contacting the solid-electrolyte.
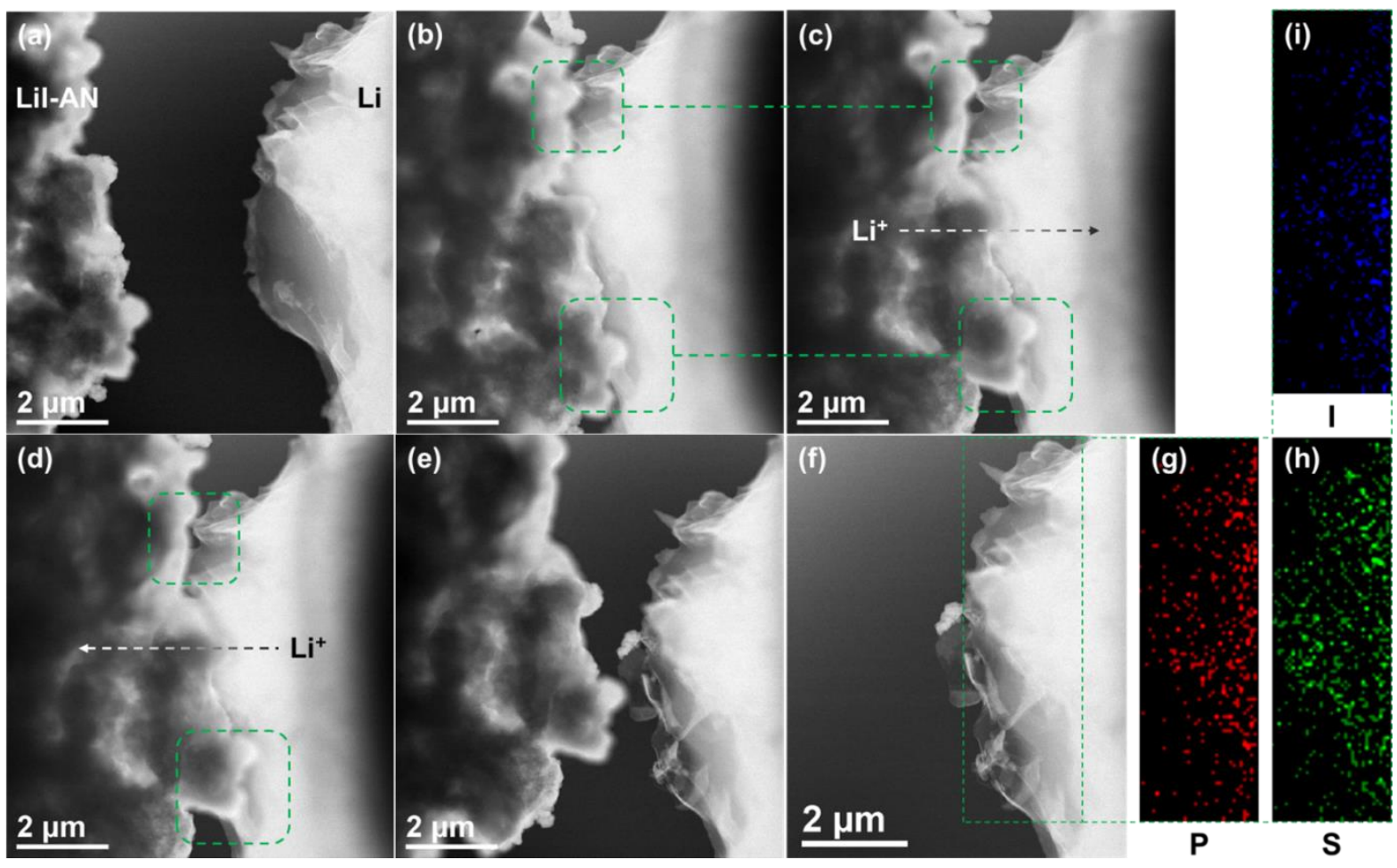

Figure 2. Figure 2: In-situ STEM images showing void formation at the interface of the solid electrolyte and Li metal during Li plating from the electrolyte (a-c), and subsequent retention of these voids upon $\mathrm{Li}$ stripping (d) with the additional formation of dead Li (e) and the detection of solid electrolyte elements on the Li metal surface via EDS (f-i).

\section{References}

[1] David Cooper, et al., Advanced Materials 29 (2017), p. 1700212.

[2] Fei-Hu, et al., ACS Nano 11 (2017), p. 8628.

[3] Ze Yang, et al., Energy Storage Materials 9 (2017), p. 214

[4] Ovidiu Cretu, et al., Nano Letters 13 (2013), p. 3487.

[5] Nikhilendra Singh, et al. Chem. Mater. 32 (2020), p 7150.

[6] Marm Dixit, et al. Matter 3 (2020), p 2138. 